\title{
ANALYSIS OF HIERARCHICAL SCHEDULING FOR HETEROGENEOUS TRAFFIC OVER NETWORK
}

\author{
Rabie Barhoun ${ }^{1}$, Abdelwahed Namir ${ }^{1}$ and Anas Barhoun ${ }^{2}$ \\ ${ }^{1}$ Department of Mathematics and Computer Science, University Hassan II, Mohammedia, \\ P.O Box 7955 Sidi Otmane, Casablanca, Morocco \\ barhounrabie@yahoo.fr and a.namir@yahoo.fr \\ ${ }^{2}$ Department of Mechanical and Aerospace Engineering, Syracuse University, 149 Link \\ Hall, Syracuse NY 13244, USA \\ abarhounesyr.com
}

ABSTRACT

Scheduling real time and non real time packets at network nodes has an important impact by reducing the processing overhead, queuing delay and response time. Most of the existing packet scheduling algorithms used in network based on First-In First-Out (FIFO), non-preemptive priority, and preemptive priority scheduling. However, these algorithms incur a large processing overhead, queuing delay and response time and are not dynamic to the data traffic changes. In this paper, we present a new hierarchical scheduling algorithm to assign priority, Hierarchical Hybrid EDF/FIFO which can not only serve the real time traffic but also provide best effort service to non real time traffic. To examine our approach for scheduling, we realized our analytical study to express the worst case queuing delay and the worst case response time for different traffics. The simulation results showed that the Hierarchical hybrid EDF/FIFO achieved the minimum packet delay and adequate loss packet for non real time traffic when compared with Hierarchical FIFO. In general, the performances of our approach draw near to Hierarchical EDF which confirms the effectiveness of this approach.

\section{KEYWORDS}

Real time traffic, Non real time traffic, Packet scheduling, Hierarchical Hybrid EDF/FIFO, Performance.

\section{INTRODUCTION}

The quality of service $(\mathrm{QoS})$ is guarantees to network applications where is capable to respond to requirements of QoS for different network nodes. Nowadays, many application of newly developed systems, such as wireless sensor networks or high speed packet switching networks, which are usually referred to as 'multi-class traffic'. This is particularly evident in the era of growing, with both real time and non real time classes of traffic, in which the quality of service (QoS) of the applications is to guarantee [1]. The requirements of Qos depend on many parameters such as the delay, bandwidth, jitter and reliability or a combination of some of them [2]. These parameters determine the QoS that the traffic requires. For example, a typical e-mail application has stringent requirements for reliability. Every bit has to be delivered correctly for a successful transmission. On the other hand, the e-mail application is insensitive to delay and jitter, and does not require a high Bandwidth. The user would not care less if the e-mail reaches its destination in a few milliseconds or a few seconds. However, for other types of applications these requirements can be completely different.

Moreover, the traffic circulating on the network is heterogeneous. Its characteristics vary from video traffic with stringent QoS requirements to the best-effort traffic requiring no guarantees. QoS guarantees can be provided in packet networks by the use of proper packet scheduling

DOI : $10.5121 /$ ijcnc. 2013.5308 
algorithms [3]. The goal of a scheduling algorithm is to select the high priority packet from the queue to be transmitted.

Network traffic can be categorized into two types: real time traffic, such as multimedia, businesscritical applications, telemedicine, and non real time traffic such as http data, e-mail application or file transfer. Recently, the major part of the traffic circulating in the Internet is of multimedia. These multimedia applications, due to the stringent delay constraints, have to meet certain QoS guarantees. The scheduling algorithm has a direct impact on the packets service which can take the form of a delay, a throughput or a system load, it is necessary to investigate a scheduling algorithm that adapts to traffic. The real time traffic is characterized by a bounded delay, but can tolerate some packet loss. The delay can be bounded by associating a deadline for each packet. Once a packet misses its deadline, it will be dropped as it is no longer useful. Therefore the main goal for any scheduling scheme for real time traffic is to deliver packets meets its deadline. Many scheduling algorithms have been proposed to meets this goal. The First In First Out (FIFO) scheduling algorithm, which is mostly used in conventional networks, is widely adopted for best-effort traffic. On the other hand, many scheduling algorithms have been proposed to proved different schemes of QoS guarantees, these algorithms includes Earliest Deadline First (EDF).

In this paper, we present an approach non-preemptive for scheduling two classes of traffic. The class-1 with the higher priority is real time and has absolute deadlines (where the absolute deadline is the interval of time between the arrival of a packet and its relative deadline). On the other hand, the lower priority class- 2 packets are non real time. The class- 1 packets are served according to EDF and FIFO, while the class-2 packets are served according to FIFO. To examine the proposed scheduler, we realized an analytical solution to express the queuing delay in queues and the response time for different traffics.

The rest of the paper has the following structure. Section 2 presents some scheduling algorithms. Section 3 describes the Hierarchical Hybrid EDF/FIFO system. The system model and analytical study is presented in Section 4. The Section 5 presents details of our design and a simulation study is developed with the corresponding results to gauge the benefits of our approach. Finally, Section 6 concludes this paper.

\section{NETWORKING SCHEDULING}

In this chapter we present the packet scheduling algorithms widely uses for scheduling real time traffic and no real time traffic. Based on this, on defines new policy for QoS guarantee to different applications.

\subsection{FIFO scheduling}

This is the classical scheduling algorithm deployed in the best-effort approach in the Internet and it is also known as FIFO (First In First Out). With this algorithm, the data are sent in the same order in which they are received. The complexity of this approach is very low and it is also very efficient to implement in hardware. It is a work-conserving algorithm and because its characteristics it has been adopted by a large number of network architectures. Unfortunately, FIFO has several limitations:

$\checkmark \quad$ It does not provide fairness;

$\checkmark$ The support to control congestion is limited. 
This kind of scheduler is not suitable for stream like multimedia traffic, because it is not able to isolate real time traffic from best effort ones. In this case, there is no way to guarantee a specific level of quality to real time traffic.

As a computer revolution, many scheduling algorithms have been proposed to meet this goal. The First-In-First-Out (FIFO) scheduling algorithm, which is mostly used in conventional networks, is widely adopted for best-effort traffic. This method is easy to realize and her complexity is reduced to $\mathrm{O}(1)$. In the other hand weak point of this method is long delay time from Queue because every packet is processed in singles a Queue. Occur delay in processing single Queue. FIFO can be used in real time system with non heavy traffic, in this way the advantage of using a simple algorithm will help in decreasing the overhead time due to the scheduler processing.

\subsection{EDF Scheduling}

Many real time systems rely on the earliest deadline first (EDF) [4] scheduling algorithm. This algorithm scheduling, represent a special attention [5], [6], [7]. Its principle is simple, that is sorting packets in order of their absolute deadline. More precisely, each traffic on a deadline, representing the constraint on the response time. Thus, when a packet arrives at a node (router or switch), the EDF scheduling algorithm assigns an absolute deadline equal to its arrival time in the node plus the relative deadline of the traffic which belongs. The packet with the smallest absolute deadline will be transmitted first. The study of the EDF scheduling is interesting, especially since this algorithm has been proved optimal uniprocessor environment where the moment's activation of the packets is not known a priori [8]. Its implementation in networks has been the subject of many studies [9] [10] and recently by [11], [12], [13], [14]. However, this algorithm has a signification complexity deriving from an incremental cost of classification packets, which increases with the queue's length, furthermore, its efficiency becomes more consistent in case of high load.

\section{Hierarchical Hybrid SCHEdUling}

The larger network such as internet offering a single best effort service in which coexist the various requirements, such as business-critical applications, telemedicine, message or file transfer. To satisfy the various requirements, a uniform treatment was inappropriate. Even with a surplus of bandwidth, the demanding traffics in real time (i.e. critical applications or response time) are not privileged. They can be penalized by lower priority traffics.

In the paper [15] we propose a new scheduling algorithm Hierarchical Hybrid EDF/FIFO which can meet the real time with complexity reduces while continuing to provide best effort service over heterogeneous network traffic. In EDF scheduler, low priority traffic, such as non real traffic, can starve as it is characterized by long lateness [16]. Despite EDF provides stable QoS guarantees to High priority traffics, such as Real-Time traffic, the deadline miss rates of the low priority traffics can be unacceptably high.

We can summarize the main drawbacks of the EDF scheduler when implemented in a large network where there are different applications, as follows:

$\checkmark$ The work complexity of the EDF scheduler is important, and which becomes more pronounced when the queue length increases drastically.

$\checkmark$ The traffic non real time still penalized by real time traffic that receives a high priority. 
The Hierarchical Hybrid EDF/FIFO [17] can serve heterogeneous traffic, by using a priority queue Hybrid EDF/FIFO for class-1, while continuing to provide best effort service class-2 by lower priority queue FIFO. The queue process control is proposed to eliminate the queue overload. That leads to the numerical results shown below which prove the differentiated service for heterogeneous traffic.

The Hybrid EDF/FIFO scheduling proposed [18] to overcome the first drawback of the EDF. This algorithm combines both the EDF and the FIFO scheduling algorithms. Idea consists in limiting the usage of EDF to the scheduling of the first $\mathrm{n}$ packets; and the remaining packets are scheduled via the simple and fast FIFO algorithm. Analyzing this scheduling algorithm, it is obvious that the last drawback is still discovered when Hybrid EDF-FIFO is used to schedule heterogeneous network traffic. Our approach Hierarchical Hybrid EDF/FIFO satisfies this objective. It can meet the real time needs of such applications, by using a priority queue Hybrid EDF/FIFO, while continuing to provide best effort service to non real time traffic by lower priority queue FIFO.

We classified the traffics into two classes; the first class (class-1) is a priority and represents real time traffics. The second class (class-2) is a lower priority and corresponds to non real time traffics. Earliest Deadline First (EDF) is on ideal scheduler for real time traffics because the optimality of this algorithm has been proved for number criteria. However, this algorithm knows a complexity that increases with the size of the queue. In order to schedule the traffic of class-1 with a complexity reduces and to not penalize the traffics of class-2, our scheduling approach is non-preemptive and based in two queues. The first is a queue hybrid EDF/FIFO for class-1 that combines the two polices First In First Out (FIFO) and EDF. The second queue for class-2 is scheduled by simple FIFO policy. The complexity of this approach is less than $O(n \log n)$. The performances of the proposed approach are evaluated by the miss deadline of the packet in the hybrid queue the class- 1 and the average time of residence of packet in the FIFO queue of class-2.

\section{SYSTEM MODEL}

In this paper, we consider the uniprocessor case which corresponds to the particular case where the system (network) consists of a single node, and the scheduling tasks (packets) are non preemptive. The system comprises a set of $\mathrm{N}$ independent real time flows $\left\{\tau_{1}, \tau_{2}, \ldots, \tau_{N}\right\}$ and $\mathrm{M}$ independent non real time flows $\left\{\tau_{1}^{\prime}, \tau_{2}^{\prime}, \ldots, \tau_{M}^{\prime}\right\}$. The packets generated by different flows are classified into two classes: The class- 1 for real time packets and the class- 2 for non real time packets. Each flow can be periodic or sporadic, and it can be, also, finite or infinite sequence of packets (jobs) that are executed on a single processor.

Each real time flow $\tau_{i}$ is defined by several parameters:

$C_{i}$ : Worst-case execution time.

$T_{i}$ : Period or minimum inter-arrival time.

$D_{i}:$ Relative deadline.

$J_{i}$ : Maximum release jitter.

$a_{i, q}$ : The arrival time of $\mathrm{q}^{\text {th }}$ packet (job).

If a packet from the real time flow $\tau_{i}$ (class-1) arrives at time $a_{i}$, then it will be released for execution at time $a_{i}+J_{i}$ and have an absolute deadline of $a_{i}+D_{i}$.

On the other hand, the non real time flow $\tau_{i}^{\prime}$ is characterized by the following four parameters: $C_{i}^{\prime}$ : Worst-case execution time. 
$T_{i}^{\prime}$ : Period or minimum inter-arrival time.

$J_{i}^{\prime}$ : Maximum releases jitter.

$a_{i, q}^{\prime}$ : The arrival time of $\mathrm{q}^{\text {th }}$ packet.

System overheads would be ignored in this study. In this model, we assume that the system is schedulable ie total worst-case utilization of the system is equal:

$$
U=U_{F I F O}+U_{E D F / F I F O}<1
$$

Where $U_{E D F / F I F O}$ and $U_{F I F O}$ are worst case utilization the Hybrid EDF/FIFO scheduling and FIFO Scheduling respectively.

In the following section, first we express the response time and the queuing delay of the packets under EDF and FIFO scheduling based on studies published in $[19,20]$. Then, we express analytically the worst-case response time and the worst-case queuing delay of the packets under our Hierarchical Hybrid EDF/FIFO scheduling.

\subsection{FIFO Scheduling}

The following results have been introduced by [18], where the start time later than the $\mathrm{q}^{\text {th }}$ packet of $\tau_{i}$ under FIFO scheduling is defined by:

$$
W_{i, q}^{F I F O}=\sum_{j \neq i, a_{i, q}+J_{j}>0}\left(1+\left\lfloor\frac{a_{i, q}+J_{j}}{T_{j}}\right\rfloor\right) \cdot C_{j}+\left\lfloor\frac{a_{i, q+} J_{i}}{T_{i}}\right\rfloor \cdot C_{i}
$$

The worst case queuing delay of the $\mathrm{q}^{\text {th }}$ packet of $\tau_{i}$ under FIFO scheduling is:

$$
\bar{W}_{i, q}^{F I F O}=W_{i, q}^{F I F O}-a_{i, q}(1)
$$

The worst case response time of the $\mathrm{q}^{\text {th }}$ packet of $\tau_{i}$ under FIFO scheduling is:

$$
R_{i, q}^{F I F O}=\bar{W}_{i, q}^{F I F O}+C_{i}
$$

In the case of the FIFO scheduling where we have exactly one flow and q-1 packets that precedes our packet, the worst case response time of the $\mathrm{q}^{\text {th }}$ packet can be written as:

\subsection{EDF scheduling}

$$
R_{i, q}^{F I F O}=\sum_{k=1}^{q} C_{k}+J_{i}
$$

The following results are from [19], where the start time later than the $\mathrm{q}^{\text {th }}$ packet of $\tau_{i}$ under EDF scheduling is defined by the following recursive equation: 


$$
\begin{gathered}
W_{i, q}^{E D F}=\left\lfloor\frac{a_{i, q+} J_{i}}{T_{i}}\right\rfloor \cdot C_{i}+\sum_{j, a_{i, q}+D_{i} \geq D_{j}-J_{j}}\left(1+\left\lfloor\frac{\min \left(a_{i, q}+D_{i}-D_{j} ; W_{i, q}^{E D F}\right)+J_{j}}{T j}\right\rfloor\right) \cdot C_{j} \\
\quad+\max \left(0, \max _{k, a_{i, q}+D_{i} \geq D_{k}-J_{k}}\left(C_{k}-1\right)\right)
\end{gathered}
$$

The worst case queuing delay of $\mathrm{q}^{\text {th }}$ packet of $\tau_{i}$ under EDF scheduling is:

$$
\bar{W}_{i, q}^{E D F}=W_{i, q}^{E D F}-a_{i, q}
$$

The worst case response time of the $\mathrm{q}^{\text {th }}$ packet of $\tau_{i}$ under EDF scheduling is:

$$
R_{i, q}^{E D F}=\bar{W}_{i, q}^{E D F}+C_{i}
$$

\subsection{Hybrid EDF/FIFO scheduling}

The hybrid queue EDF/FIFO consists of applying the EDF algorithm to $\mathrm{k}$ first packets of the queue, and the FIFO algorithm to the remaining packets. In fact, if the $\mathrm{q}^{\text {th }}$ packet arrives at Hybrid queue EDF/FIFO at $a_{i, q}$ time, it will be considered by EDF at the following time:

$$
a_{i, q}+\bar{W}_{i, q}^{F}
$$

And $\bar{W}_{i, q}^{F}$ is the worst case queuing delay of the $\mathrm{q}^{\text {th }}$ packet of $\tau_{i}$ introduced by FIFO scheduling in Hybrid queue EDF/FIFO.

We can define the worst case queuing delay of $\tau_{i}$ introduced by FIFO scheduling in Hybrid queue EDF/FIFO by:

$$
\bar{W}_{i}^{F}=\max _{q} \bar{W}_{i, q}^{F}(5)
$$

From (4) and (5), we can define the maximum release jitter of $\tau_{i}$ which be considered by EDF scheduling in Hybrid queue EDF/FIFO by:

$$
J_{i}^{*}=\bar{W}_{i}^{F}(6)
$$

From (2), (4) and (6) the start time later than the $\mathrm{q}^{\text {th }}$ packet of $\tau_{i}$ under hybrid EDF/FIFO scheduling is:

$$
\begin{aligned}
W_{i, q}^{E / F}=\left[\frac{a_{i, q}+J_{i}^{*}}{T_{i}}\right\rfloor \cdot C_{i}+\sum_{j, a_{i, q}^{*}+D_{i} \geq D_{j}-J_{j}}\left(1+\left\lfloor\frac{\min \left(a_{i, q}+D_{i}-D_{j} ; W_{i, q}^{E / F}\right)+J_{j}^{*}}{T_{j}}\right]\right) \cdot C_{j} \\
\quad+\max \left(0, \max _{k, a_{i, q}^{*}+D_{i} \geq D_{k}-J_{k}}\left(C_{k}-1\right)\right)(7)
\end{aligned}
$$

From (4), (6) and (7) the worst case queuing delay of the $\mathrm{q}^{\text {th }}$ packet of $\tau_{i}$ under hybrid EDF/FIFO is defined by: 
International Journal of Computer Networks \& Communications (IJCNC) Vol.5, No.3, May 2013

$$
\bar{W}_{i, q}^{E / F}=W_{i, q}^{E / F}-a_{i, q}(8)
$$

The worst case response time of the $\mathrm{q}^{\text {th }}$ packet of $\tau_{i}$ under hybrid EDF/FIFO scheduling is:

$$
R_{i, q}^{E / F}=\bar{W}_{i, q}^{E / F}+C_{i}
$$

\subsection{Hierarchical hybrid EDF/FIFO scheduling}

From (7), we can express the worst case queuing delay of $\mathrm{q}^{\text {th }}$ packet class- 1 of $\tau_{i}$ as follows:

$$
\bar{W}_{i, q}^{h(E / F)}=\bar{W}_{i, q}^{E / F}+\max _{j=1, M} C_{j}^{\prime}
$$

Where $\max _{j=1, M} C_{j}^{\prime}$ is the worst time of blockage introduced by the FIFO scheduling.

The worst case response time of $\mathrm{q}^{\text {th }}$ packet class- 1 of $\tau_{i}$ is:

$$
R_{i, q}^{h(E / F)}=\bar{W}_{i, q}^{h(E / F)}+C_{i}
$$

From (1) and (4) the worst case queuing delay of $\mathrm{p}^{\text {th }}$ packet class- 1 of $\tau_{j}^{\prime}$ as follows:

$$
\bar{W}_{j, p}^{h(F)}=\bar{W}_{j, p}^{F I F O}+\bar{W}_{l}^{E / F}
$$

Where $\bar{W}_{l}^{E / F}$ is the worst time of blockage introduced by the last packet in Hybrid queue EDF/FIFO.

The response time of $\mathrm{p}^{\text {th }}$ packet class- 2 of $\tau_{j}^{\prime}$ is defined by:

$$
R_{j, p}^{h(F)}=\bar{W}_{j, p}^{h(F)}+C_{j}^{\prime}
$$

\section{SySTEM QUEUE MANAGEMENT}

Our Hierarchical Hybrid EDF/FIFO system consists of two queues: A high priority queue with Hybrid EDF/FIFO service for the real-time traffic such as video, voice and Multimedia, and a low priority queue with FIFO service for the non-real-time traffic such as http data. Table 1 summarizes the internal parameters of our scheduling policy. 
International Journal of Computer Networks \& Communications (IJCNC) Vol.5, No.3, May 2013

Table 1. Internal parameter of the Hierarchical Hybrid EDF/FIFO algorithm

\begin{tabular}{|c|l|}
\hline Parameter & \multicolumn{1}{c|}{ Signification } \\
\hline$H$ & The Hybrid EDF/FIFO queue \\
\hline$H_{e}$ & The portion of the H queue managed by EDF \\
\hline$H_{f}$ & The portion of the H queue managed by FIFO \\
\hline$F$ & Tthe queue FIFO \\
\hline$L$ & Length of the Hierarchical Hybrid EDF/FIFO queue \\
\hline$x$ & Type of the queue (service) H or F \\
\hline$L_{x}$ & Length of the x queue \\
\hline$k$ & Length of the x queue managed by EDF \\
\hline$n$ & Length of the x queue managed by FIFO \\
\hline$q_{x}$ & Number of packet in $\mathrm{x}$ queue \\
\hline$p$ & Packet \\
\hline$p^{x}$ & Packet of the head of $q_{x}$ \\
\hline$p_{x}$ & Packet of the tail of $q_{x}$ \\
\hline$d_{p}$ & Relative Deadline of the packet $\mathrm{p}$ \\
\hline
\end{tabular}

The algorithm for serving the Hierarchical Hybrid EDF/FIFO Queue:

$\mathrm{p} \leftarrow$ received packet;

$\mathrm{x} \leftarrow$ class of $\mathrm{p}$; $(\mathrm{H}$ or $\mathrm{F})$

If $q_{H}+q_{F}+1<L$

discard $\mathrm{p}$;

- The process of inserting the packet $\mathrm{p}$ in the Hybrid EDF/FIFO queue

if $q_{H}+1<L_{H}$

if $d_{\mathrm{HE}}<d_{p}$

insert $\mathrm{p}$ to $\mathrm{H}_{\mathrm{e}}$

else if $a_{p H \varepsilon}<a_{p}$ insert $\mathrm{p}_{\mathrm{He}}$ to $\mathrm{H}_{\mathrm{f}}$

insert $p$ to $\mathrm{H}_{\mathrm{e}}$

else insert $p$ to $\mathrm{H}_{\mathrm{f}}$

else discard $\mathrm{p}$;

- The process of inserting the packet $\mathrm{p}$ in the queue $\mathrm{F}$

if $q_{F}+1<L_{F}$

else discard $\mathrm{p}$;

insert $\mathrm{p}$ to $\mathrm{F}$ 
- The process of serving

If $q_{H}=0$

/* The F queue well be served */

else

$$
\begin{aligned}
& q_{F} \leftarrow q_{F}-1 ; \\
& \text { Send }\left(\mathrm{p}_{\mathrm{F}}\right) ; \\
& q_{F} \leftarrow q_{F}-1
\end{aligned}
$$

/* The H queue well be served */

$$
\begin{aligned}
& q_{H} \leftarrow q_{H}-1 ; \\
& \operatorname{Send}\left(\mathrm{p}_{\mathrm{H}}\right) ;
\end{aligned}
$$

We consider a network topology comprising 4 CBR periodic traffic sources, three of them generate real time traffic (class-1) while the fourth generates non real time traffic (class-2), sharing equally a $3 \mathrm{Mbps}$ link. The link is managed by three scheduling algorithms Hierarchical Hybrid EDF/FIFO, Hierarchical EDF and Hierarchical FIFO. The Buffer size is set to 100 packets for class-1 and 100 packets for class-2.

The packet size was chosen to be of 1150 Byte for all class, the reason to choose this value is that almost $50 \%$ of the traffic being propagated across the internet has a packet size of 1150 Byte as shown in Figure 4 which was explored by the research work carried out in [21].

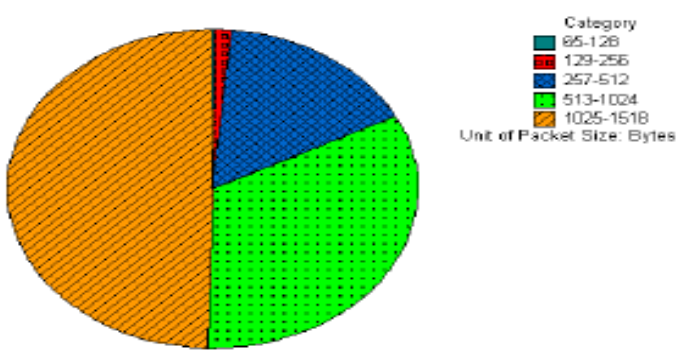

Figure 1. Packet size distribution

For the three traffics of class- 1 , we consider three flowing deadlines: $(d 1=3 \mathrm{~ms} ; \mathrm{d} 2=50 \mathrm{~ms}$; $\mathrm{d} 3=120 \mathrm{~ms}$ ). For all traffic we used periodic CBR traffic.

Different graphs are plotted below to compare the performance of the three scheduling algorithms: Hierarchical FIFO, Hierarchical EDF and Hierarchical Hybrid EDF/FIFO for the different traffic (class- 1 and class-2). Figures 2 and 3 show the packet percentage miss deadline of the class-1 (for examples video, Voice and telemedicine) when using each the three scheduling algorithm. The results show that number of packets whose deadlines are met increases drastically when the size of the EDF part of the queue Hierarchical Hybrid EDF/FIFO increases. So, the number of packet miss deadline of our approach is less than the ones of the approach Hierarchical FIFO, but it approximates the ones of the Hierarchical EDF proportionately with size of the EDF's hierarchical Hybrid EDF/FIFO. 
International Journal of Computer Networks \& Communications (IJCNC) Vol.5, No.3, May 2013

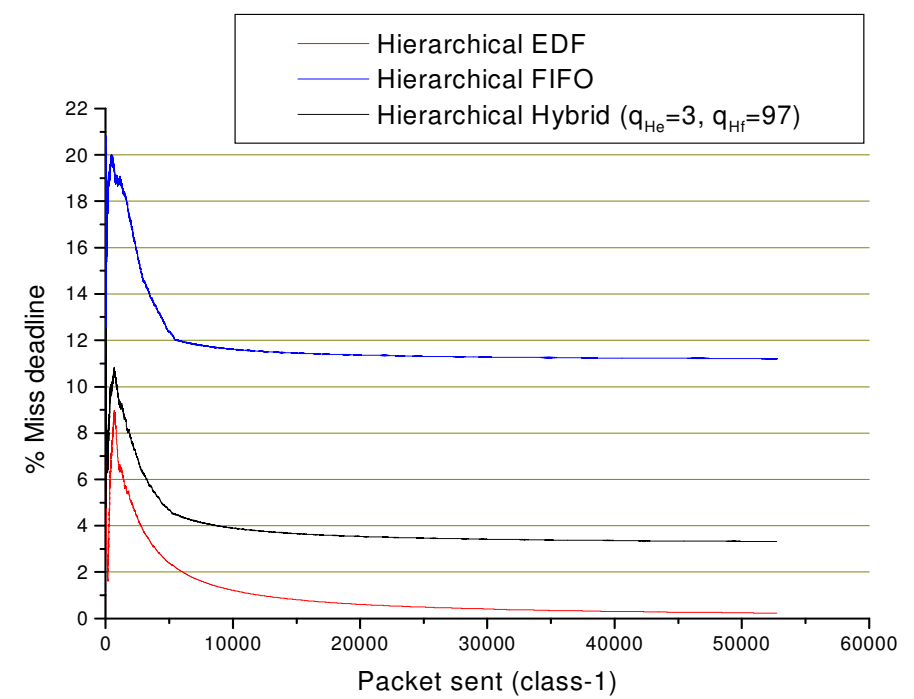

Figure 2. Comparison Hierarchical FIFO, Hierarchical EDF and Hierarchical Hybrid EDF/FIFO with $\mathrm{q}_{\mathrm{He}}=3$ and $\mathrm{q}_{\mathrm{Hf}}=97$

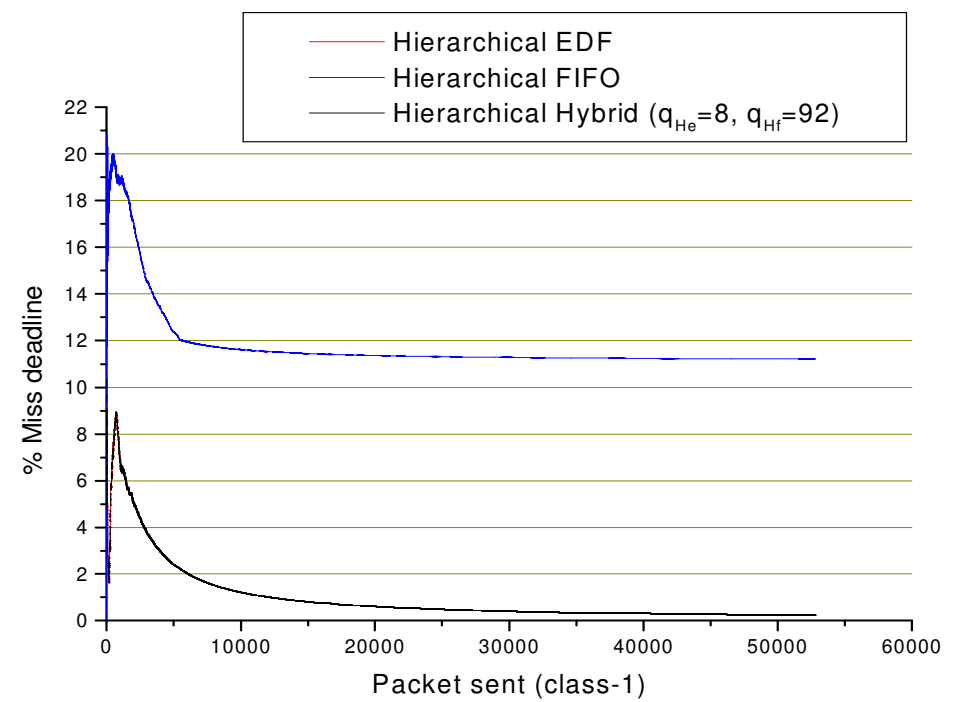

Figure 3. Comparison Hierarchical FIFO, Hierarchical EDF and Hierarchical Hybrid EDF/FIFO with $\mathrm{q}_{\mathrm{He}}=8$ and $\mathrm{q}_{\mathrm{Hf}}=92$ 

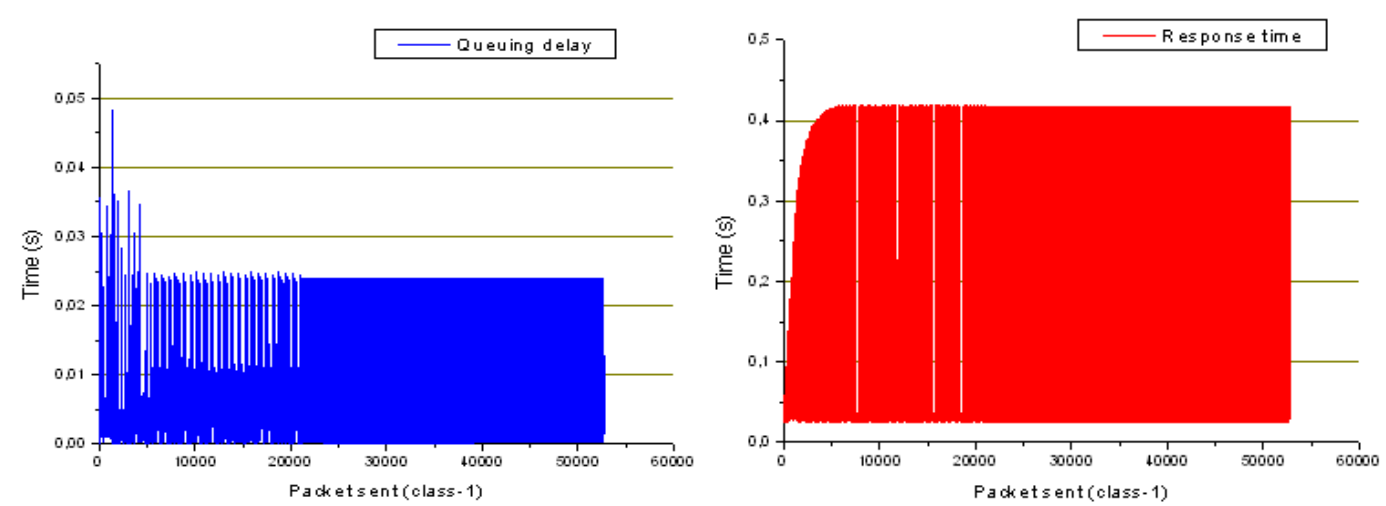

Figure 4. Queuing delay and Response time of class-1 for the Hierarchical EDF scheduling
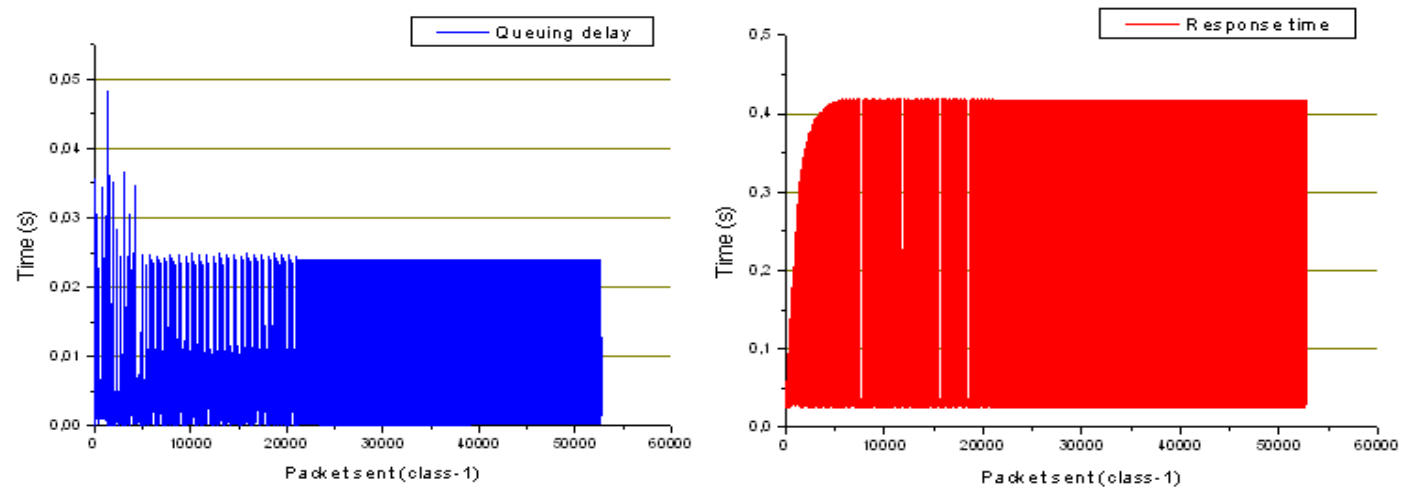

Figure 5. Queuing delay and response time of class-1 for the Hierarchical Hybrid $\left(\mathrm{q}_{\mathrm{He}}=8, \mathrm{q}_{\mathrm{Hf}}=92\right)$ scheduling

Figures 4 and 5 shows that the queuing delay for the class- 1 packets of the two algorithms Hierarchical EDF and Hierarchical Hybrid $\left(\mathrm{q}_{\mathrm{He}}=8, \mathrm{q}_{\mathrm{Hf}}=92\right)$ is almost identical. We have the same remark for the response time (Figures 4 and 5).
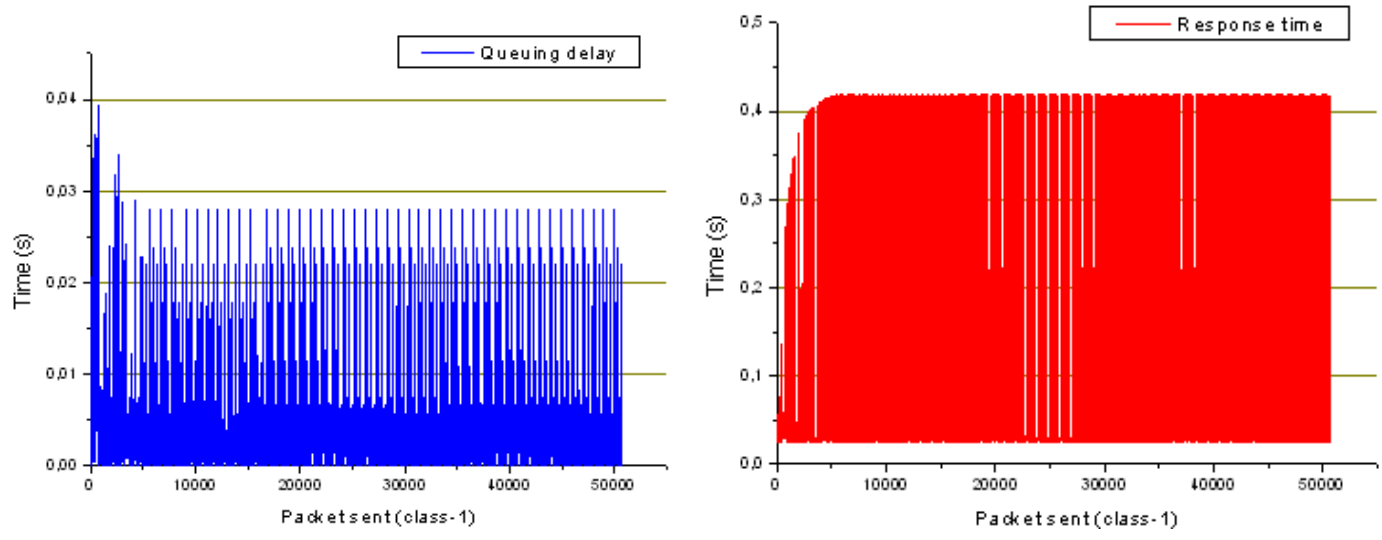

Figure 6. Queuing delay and response time of class- 1 for the Hierarchical hybrid $\left(\mathrm{q}_{\mathrm{He}}=3, \mathrm{q}_{\mathrm{Hf}}=97\right)$ scheduling 
Figures 6 and 7 show that the number of class-1 packets served by Hierarchical Hybrid $\left(\mathrm{q}_{\mathrm{He}}=3, \mathrm{q}_{\mathrm{Hf}}=97\right)$ technique is slightly more important than the number of packets served by pure FIFO. The queuing delay of class-1 packets served by hierarchical hybrid $\left(\mathrm{q}_{\mathrm{He}}=3, \mathrm{q}_{\mathrm{Hf}}=97\right)$ algorithm varies between $0 \mathrm{~s}$ and $0.039 \mathrm{~s}$ and the response time between $0.025 \mathrm{~s}$ and $0.410 \mathrm{~s}$, whereas, for the Hierarchical FIFO the queuing delay varies between $0 \mathrm{~s}$ and $0.148 \mathrm{~s}$ and the response time between $0.030 \mathrm{~s}$ and $0.042 \mathrm{~s}$. The queuing delay of almost all class- 1 packets served by Hierarchical FIFO scheduling is less than that served by Hierarchical Hybrid $\left(\mathrm{q}_{\mathrm{He}}=3, \mathrm{q}_{\mathrm{Hf}}=97\right)$.
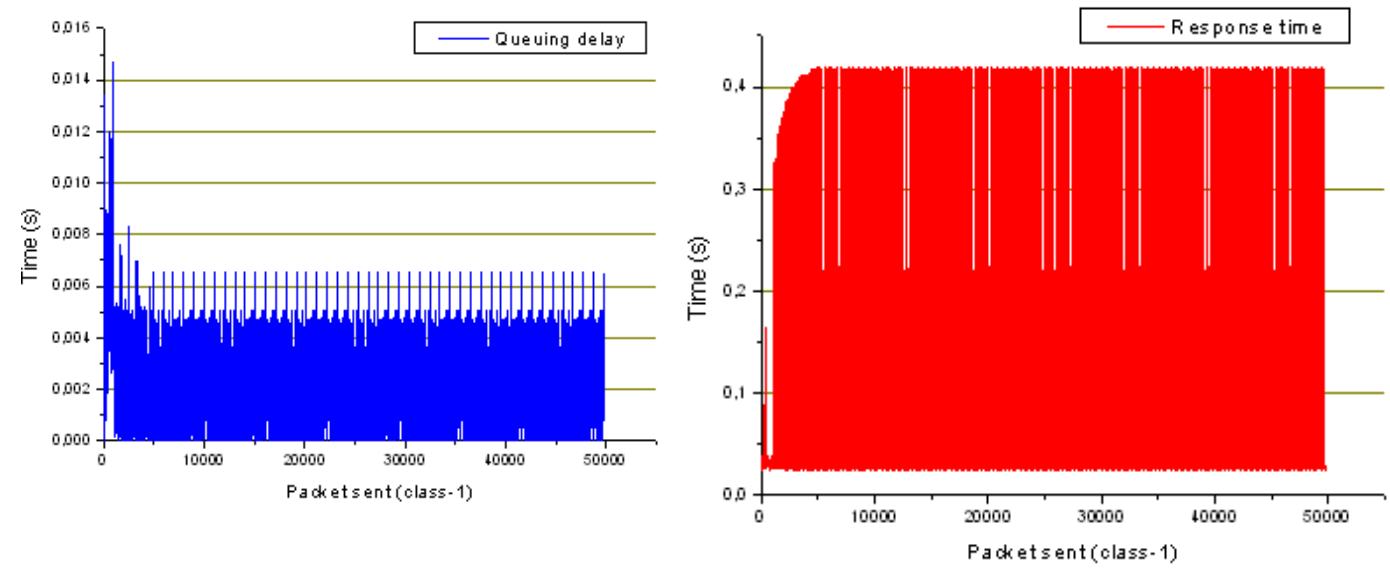

Figure 7. Queuing delay and Response time of class-1 for the Hierarchical FIFO scheduling

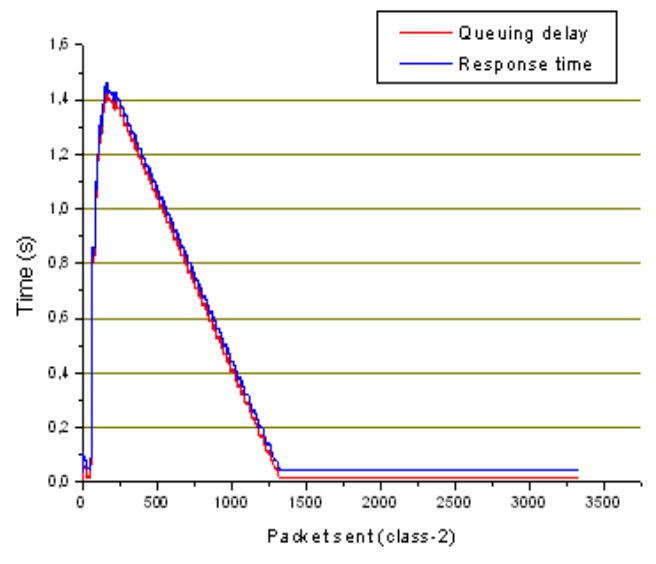

Figure 8. Response time and queuing delay of class- 2 for the Hierarchical EDF scheduling

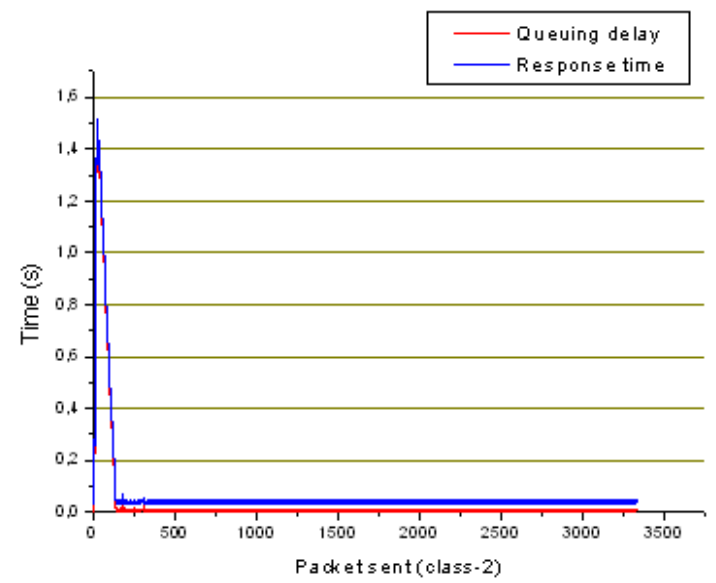

Figure 9. Response time and queuing delay of class-2 for the Hierarchical FIFO scheduling

Figures 8 and 9 show that the queuing delay and response time of the class-2 packets with the two scheduling algorithms Hierarchical EDF and Hierarchical FIFO are different. 


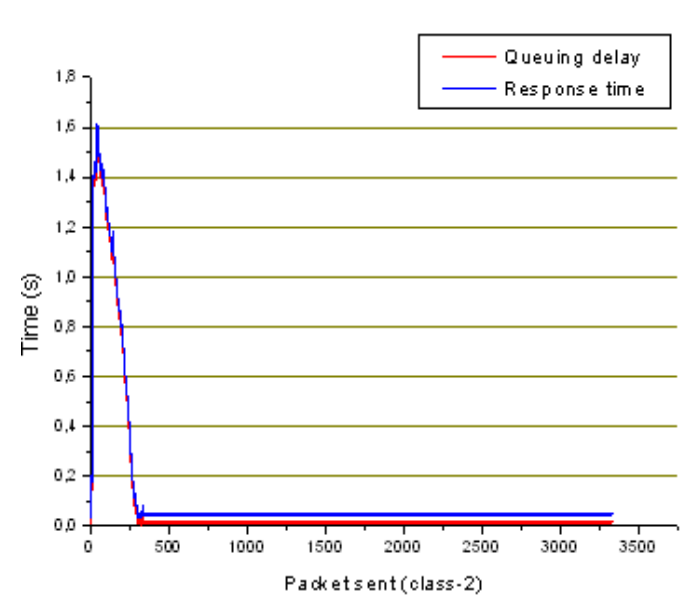

Figure 10. Queuing delay and response time of class- 2 for the Hierarchical hybrid $\left(\mathrm{q}_{\mathrm{He}}=3, \mathrm{q}_{\mathrm{Hf}}=97\right)$ scheduling

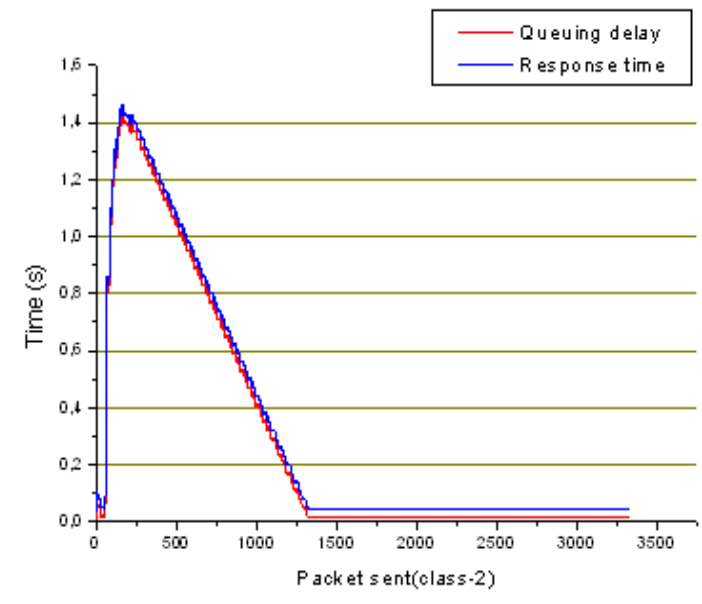

Figure 11. Queuing delay and response time of class- 2 for the Hierarchical hybrid $\left(\mathrm{q}_{\mathrm{He}}=8, \mathrm{q}_{\mathrm{Hf}}=92\right)$ scheduling

Figures 10 and 9 show that the queuing delay and response time of the class-2 packets of two scheduling algorithms Hierarchical Hybrid $\left(\mathrm{q}_{\mathrm{He}}=3, \mathrm{q}_{\mathrm{Hf}}=97\right)$ and Hierarchical FIFO are almost similar. We can notice the same similarity between the Hierarchical Hybrid $\left(\mathrm{q}_{\mathrm{He}}=8, \mathrm{q}_{\mathrm{Hf}}=92\right)$ and Hierarchical EDF (Figures 11 and 8). In conclusion, the behavior of our scheduling Hierarchical Hybrid EDF/FIFO when serving class-2 packets approximates to behavior of the Hierarchical Hybrid EDF when the size of the EDF part of the queue Hierarchical Hybrid EDF/FIFO increases.

Finally, by analyzing the different figures above, we can assert that the Hierarchical Hybrid EDF/FIFO scheduling shows a better performance of serving packet over heterogeneous network traffic with reduced complexity.

\section{Conclusions}

In this paper we presented a new scheduling approach for two traffic class. The prioritized class-1 packets are considered to be real-time and served according to the hybrid policy scheduling $\mathrm{EDF} / \mathrm{FIFO}$, and the non real time class-2 packets corresponds to a lower priority and served by FIFO policy. The service discipline of the system is non-preemptive. We realized our analytical study to express the worst-case queuing delay and the worst-case response time for different traffics. The main advantages of our hierarchical Hybrid EDF/FIFO queue resides in reducing the work complexity of the EDF algorithm, while maintaining to a great extent EDF's advantage in regarding packets' deadline respect while continuing to provide best effort service over heterogeneous network traffic environment. The simulation results showed that the Hierarchical Hybrid EDF/FIFO scheduler produces a better performance of packet serving over heterogeneous network traffic by achieving the minimum miss deadline's the packets. 


\section{REFERENCES}

[1] H. Zhang "Service, (1995) "disciplines for guaranteed performance service in packet switching networks", proceedings of the IEEE, 83(10):1374-1399.

[2] Andrew S. Tanenbaum, (2003) "Computer Networks", Fourth Edition, Pearson Education.

[3] P.Goyal, H.M.Vi, and H.Chen, (1996) "Start-time Fair Queuing: A scheduling algorithm for integrated services", proceedings of the ACM-SIGCOMM '96, Palo Alto, CA, pp 157-168.

[4] Liu, C.L. and Layland, J.W. (1973) "algorithms for multiprogramming in a hard real-time environment", Journal of the ACM, 20(1), pp 46-61.

[5] K. Jeffay, D. Stanat, and C. Martel, (1991) "On non-preemptive scheduling of periodic and sporadic tasks", In IEEE Real-Time Systems Symposium, pages 129-139.

[6] J. Migge and A. Jean-Marie, (1998) "Timing analysis of real-time scheduling policies: A trajectoy based model”, In Tech. Report, INRIA, RR-3561.

[7] F. Chiussi, M. Gerla, and V. Sivaraman, (2000) "Traffic shaping for end-to-end delay guarantees with edf scheduling”, In proceedings of International Workshop on Quality of Service (IWQoS).

[8] Towsley, D. and Panwar, S.S. (1991) "Optimality of the Stochastic Earliest Deadline Policy for the G/M/c Queue Serving Customers with Deadlines". Tech. Report 91-61, Univ. Massachusetts.

[9] R. Chipalkatti, J. Kurose, and D. Towsley, (1989) "Scheduling Policies for Real-Time and NonReal-Time Traffic in a Statistical Multiplexer”, In Proceedings of INFOCOM'89, pages 774783

[10] J. Peha and F. Tobagi, (1990) "Evaluation scheduling algorithms for traffic with heterogeneous performance objectifs", In Proceedings of GlobeCom'90, pages 21-27 vol.1.

[11] V. Sivaraman, F. M. Chiussi, and M. Gerla, (2001) "End-to-end statistical delay service under GPS and EDF scheduling: A comparison study”, In Proceedings of IEEE INFOCOM'01, pages $1113-1122$.

[12] A. Grilo, M. Macedo, and M. Nunes, (2003) "A scheduling algorithm for QoS support in IEEE802.11e networks", In IEEE Wireless Communications, pages 36-43 vol.10.

[13] M. Andrews, (2000) "Probabilistic end-to-end delay bounds for earliest deadline first scheduling", In Proceedings of IEEE INFOCOM'00, pages 603-612.

[14] M. D. Natale and A. Meschi, (2001) "Scheduling messages with earliest deadline techniques", In Journal Real-Time Syst., pages 255-285 vol.20.

[15] R. Barhoun, A. Namir, (2011) "Packet scheduling of two classes flow" in International Journal of Computer Science \& Information Technology (IJCSIT) Vol 3, No 4.

[16] F. Zhu, J.P. Lehoczky, J.P. Hansen, R. Rajkumar, (2005) "Diff-EDF: A Simple Mechanism for Differentiated EDF Services", Proceedings of the 11th IEEE Real-Time and Embedded Technology and Applications Symposium, pp. 268-277.

[17] R. Barhoun, A. NAMIR, (2012) "Hierarchical scheduling for heterogeneous flows" IEEE International Conference on Multimedia Computing and Systems (IEEE ICMCS'12), pages 640 644.

[18] O. C. Saadbouh, W. Fawaz and K. CHEN, (2006) "A Novel Approach for Real Time Flows Scheduling”, IEEE Globecom 2006, San Francisco, California, USA.

[19] K. Tindell, A. Burns, and A. J. Wellings, (1994) "Calculating Controller Area Network (CAN) message response times" IFAC DCCS'94, Toledo, Spain.

[20] L. George, N. Rivierre, and M. Spuri, (1996) "Preemptive and non-preemptive scheduling realtime uniprocessor scheduling” INRIA Research Report, No. 2966.

[21] P. Calyam and C.G. Lee, (2005) "Characterizing Voice and Video Traffic Behaviour over the Internet". In International Symposium on Computer and Information Sciences (ISCIS), Istanbul, Turkey. 